\title{
Poincare Çizimi Ölçümlerinden Topluluk Öğrenmesi Yöntemleri Kullanılarak Proses Kontrol Sistemlerinde Arıza Tespit ve Teşhisi
}

\author{
Emre Çancıoğlu ${ }^{*}$, Savaş Şahin ${ }^{2}$, Yalçın İşler ${ }^{3}$

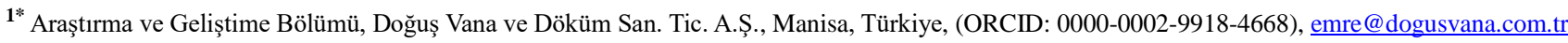 \\ ${ }^{2}$ Katip Çelebi Üniversitesi, Mühendislik ve Mimarlık Fakültesi, Elektrik ve Elektronik Mühendisliği Bölümü, İzmir, Türkiye (ORCID: 0000-0003-2065-6907), \\ phd.savas.sahin@gmail.com \\ ${ }^{3}$ Katip Çelebi Üniversitesi, Mühendislik ve Mimarlık Fakültesi, Biyomedikal Mühendisliği Bölümü, İzmir, Türkiye (ORCID: 0000-0002-2150-4756), \\ islerya@yahoo.com
}

(3rd International Congress on Human-Computer Interaction, Optimization and Robotic Applications June 11-13, 2021)

(DOI: 10.31590/ejosat.952761)

ATIF/REFERENCE: Çancioğlu, E., Şahin, S., İşler, Y. (2021). Poincare Çizimi Ölçümlerinden Topluluk Öğrenmesi Yöntemleri Kullanılarak Proses Kontrol Sistemlerinde Arıza Tespit ve Teşhisi. Avrupa Bilim ve Teknoloji Dergisi, (26), 30-34.

$\ddot{O} \mathbf{z}$

$\mathrm{Bu}$ çalışmada, farklı kimyasal birimlere ait doğrusal olmayan süreçler içeren bir endüstriyel tesisteki 20 farklı arızanın tespiti ve sınıflandırılması yapılmıştır. Kullanılan veri seti büyük bir endüstriyel tesisten elde edilen IEEEDataPort çevrimiçi veri kümesidir. Tennessee Eastman Süreci olarak bilinen bu veri seti 20 farklı hata türü ile 52 işlem noktasından alınan ölçümleri içerir. Bu ölçümler üzerinden Poincare çizimleri elde edilerek her işlem noktası için sık kullanılan doğrusal olmayan öznitelikler çıkarılmışır. Bu öznitelikler \%5 istatistiksel anlamlılık düzeyinde tek yönlü ANOVA testine uygulanarak hata türleri arasında istatistiksel olarak anlamlı fark olduğunu gösterenler seçilmiştir. Hem tüm öznitelikler hem de sadece ANOVA ile seçilen öznitelikler beş farklı topluluk öğrenmesi algoritması (Boosted Trees, Bagged Trees, Subspace Discriminant, Subspace KNN ve RUSBoosted Trees) kullanılarak sınıflandırılmıştır. Bu çalışmada elde edilen en yüksek sınıflandırıcı doğruluğu Subspace Discriminant algoritması kulanılarak \%89,5 olarak elde edilmiştir. Aynı verisetini kullanan benzer çalışmalarla kıyaslanabilir bir başarı düzeyine ulaşılmıştır. Öte yandan, ANOVA tabanlı öznitelik seçiminin bu tür endüstriyel proses tesislerinde arızaların teşhisinde bariz bir üstünlük sağlamadığı görülmüştür.

\section{Fault Detection and Diagnosis on Process Control Systems Using Ensemble Learning Algorithms from Poincare Plot Measures}

\begin{abstract}
This study aimed to detect and classify 20 different malfunctions in an industrial facility that involves nonlinear processes from various chemical units. The IEEEDataPort online dataset, acquired from a large industrial plant, was used in this study. It contains measures from 52 process points in Tennessee Eastman Process with 20 different fault types. We extracted two commonly used nonlinear features from Poincare Plots for each measurement point. The statistically meaningful features, which show statistically significant differences among fault types with a significance of 5\%, were selected from these features. Five distinct Ensemble Learner algorithms (Boosted Trees, Bagged Trees, Subspace Discriminant, Subspace KNN, and RUSBoosted Trees) discriminated the fault types using all features and the selected features only. The maximum classifier accuracies were $89.5 \%$ for both feature sets using the Subspace Discriminant method in this study. This performance is a comprehendible result among the results achieved in similar
\end{abstract}

*Sorumlu Yazar: emre@dogusvana.com.tr 
studies. On the other hand, ANOVA-based feature selection didn't result in a clear advantage to diagnose faults in such industrial process plants.

Keywords: Tennessee Eastman Process System, Fault Detection, Fault Diagnosis, Ensemble Learning, Poincare Plot Measures, OneWay ANOVA Test.

\section{Giriş}

Son yıllarda, üretim kalitesini ve sistemin performansını artırmak için daha karmaşık endüstriyel süreçlerin tasarımı zorunlu hale gelmiştir. $\mathrm{Bu}$ zorlu işlemlerin üstesinden gelebilmek için çeşitli endüstriyel ekipmanların süreci (veya süreçleri) izlemesi ve yapay zeka dahil olmak üzere ileri düzey otomasyon yöntemlerinin birlikte kullanımı ile büyük bir başarı sağlanmıştır [1]. Gelişmiş üretim süreçlerindeki ilerlemelerle birlikte, bu süreçlerin güvenilirliğini, verimliliğini ve tutarlılığını artırmak için sistem hatalarının zamanında teşhis ve tespiti oldukça önemlidir [2].

Genel amaçlı bir arıza tespit ve teşhis sistemi üç ana bölümden oluşmaktadır: (i) İzlenmekte olan sistemindeki bazı arızaların meydana geldiğini gösteren arıza tespiti, (ii) Meydana gelen arızanın türünü ve/veya yerini belirleyen arıza teşhisi ve (iii) Arızanın büyüklügünü belirleyen arıza tanımı [3]. Böyle bir sistemin çıkışı genellikle sadece arızalı veya arızasız şeklindedir. Sistemin girişi ise farklı sensörlerden gelen ölçümler ve/veya bu ölçümlerden hesaplanan öznitelikler olmaktadır. Bu sistem, verilerden ilgili modeli ya da kalıbı bulmak ve bunları görev odaklı bilgiye dönüştürmek için istatiksel tabanlı özellik seçimini, makine öğrenmesi algoritmalarıyla model oluşturulmasına dayanmaktadır.

$\mathrm{Bu}$ çalışmada, izlenen değişkenlerin fazla olduğu, kimya endüstrisinde önemli yer tutan yer alan ve literatürdeki birçok çalışmanın odak noktası olan Tennese Eastman Process (TEP) örnek alınmıştır [4, 5]. 2010 yılında gerçekleştirilen bir çalışmada, Genetik Algoritma tarafindan desteklenen Ana Bileşen Analizi (PCA) ve çok katmanlı algılayıcı (MLP) sınıflandırıcısı kullanılmıştır [6]. Aynı veri setinin analizi için başka vbir çalışmada, Kernel Tabanlı Ana Bileşen Analizi (KPCA) ve Fisher diskriminant analizi sinıflandırıcısı kullanılmıştır [7]. Bu çalışmalara ait sınıflandırıcı performansları rapor edilmemiştir. Yakın tarihli bir çalışmada, Poincare çiziminden elde edilen öznitelikler kullanılarak k-yakın komşu sınıflandırıcısı ile \%55 doğrulukla hata sınıflandırması yapılabilmiştir [10]. Daha kapsamlı başka bir çalışmada ise, destek vektör makineleri (SVM), PCA uygulanmış SVM ve çok katmanlı algılayıcı (MLP) sinıflandırıcıları kullanılarak sırasıyla $\% 49,7, \% 67,3$ ve \%73,8 sinıflandırıcı doğruluklarına ulaşılabilmiştir [11]. Bu az sayıdaki örnek çalışmadan da anlaşılacağı üzere, daha farklı sınıflandırıcı ve öznitelik çıkarma ve öznitelik seçimi algoritmalarının kullanımının araştııılmasına, böylece daha yüksek doğrulukla hata sinıflandırması yapılmasına ihtiyaç duyulmaktadır.

Bu çalışmada, sensörlerden gelen verilerden çizilen Poincare grafiklerinden hesaplanan doğrusal olmayan özniteliklerin ve bunların arasından ANOVA tabanlı istatistiksel anlamlılık ile seçilen özniteliklerin Topluluk Öğrenmesi sınıflandırıcı algoritmalarınıa uygulanmasıyla, daha yüksek doğrulukla hata tespiti yapılıp yapılamayacağı araştırılmıştır.

\section{Materyal ve Metot}

\subsection{Veri Seti}

Tennessee Eastman Process veri seti, 4 reaktif, 2 ürün, 1 yan ürün ve 1 inert bileşen olmak üzere toplam 8 kimyasal bileşenden oluşmaktadır [20]. Bu kimyasal süreçten basınç, seviye, mol oranı, sıcaklık, akış hızı ve kompresör güç çıktıları gibi sistemin özelliklerini kaydeden 52 farklı değişken elde edilmektedir. Bunlardan 11 tanesi operatörün kimyasal prosesin kontrol altında çalışmasını sağlamak için kontrol edebileceği akış hızı, karıştırıcı hızı ve valf pozisyonu gibi manipüle edilmiş değişkenlerdir.

Veri setindeki değişkenler, farklı veri dosyalarına karşılık gelen "Faulty_Free_Training", "Faulty_Free_Testing", "Faulty_Testing" ve "Faulty_Training" olarak adlandırılmıştır. Her bir veri dosyası 55 adet sütun veri içermektedir. Bunlardan "faultNumber" sütunu veri setindeki 1 ila 20 arasındaki arıza çeşidini (0 ise arıza olmaması durumunu) temsil etmektedir. "Sample" sütunu eğitim veri setinde 1 ile 500 arasında (test veri setinde ile 1 ile 960 arasında) alınan ölçümün sıra numarasını vermektedir. "xmeas_1" ile başlayıp "xmv_11" ile sonlanan (4 ila 55 arasındaki) sütunlar ise sensörlerden kayıt edilen değerleri vermektedir. Bu değerler her 3 dakikada bir kayıt edilen (yani örneklenen) eğitim veri setindeki 25 saatlik ve test veri setindeki 48 saatlik sensör verilerine karşılık gelmektedir.

$\mathrm{Bu}$ veri setinin normal olmayan koşulları tespit etme algoritmalarını karşılaştırmak için bu kadar yaygın olarak kullanılmasının ana nedeni, hatalı ve hatasız veri dosyası içermesidir. Teorik olarak, iyi bir normal olmayan koşulları tespit etme algoritması, hatasız veri kümesi için herhangi bir yanlış pozitif vermemeli ve aynı zamanda hatalı veri kümesinde ortaya çıkan 20 hatanın olabildiğince çoğunu yakalamalıdır.

\section{2. Öznitelik Çıkarma}

Doğrusal olmayan dinamiklerden alınan bir teknik olan Poincaré grafiği, x eksenindeki her verinin y eksenindeki sonraki verilere karşı çizilmesiyle oluşmaktadır [16,17]. Bu grafikte dağılımın genel şekli, zaman serilerinin dinamiklerini karakterize etmek için kullanılır. Poincaré grafiği, ham veri kümesi kullanılarak çizilir. Poincaré planının şekline bir elips yerleştirmek giderek daha popüler bir teknik haline gelmektedir [18]. Grafikteki noktaların mesafesinin standart sapması, elipsin genişliğini (SD1) ve uzunluğunu (SD2) temsil etmektedir. Şekil 1 'de örnek bir Poincare çizimi görülmektedir. 


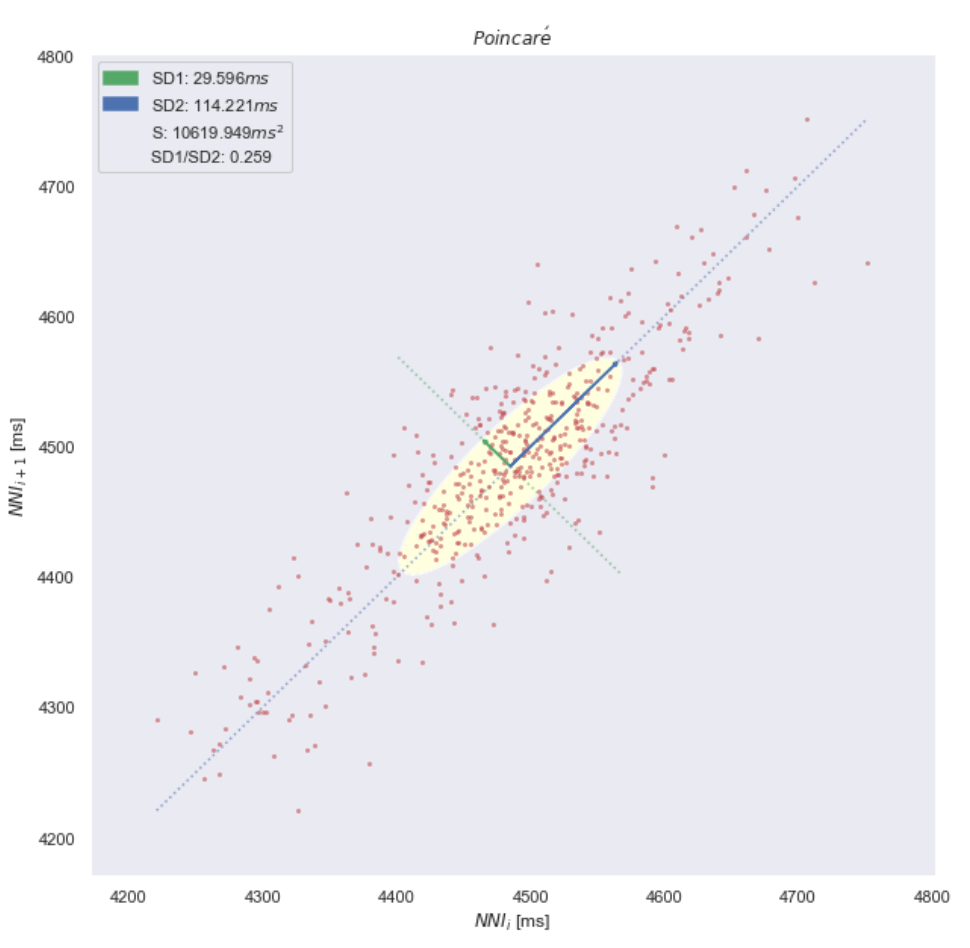

Şekil 1. Veri setindeki simulationRun=1, faultNumber=1 için xmeas_3 değerine ait Poincare çizimi ve SD1-SD2 değerleri

Poincare ölçümleri SD1 ve SD2 olup aşağıdaki formül (1) ve (2) ile hesaplanmaktadır [17,19]. Burada, SDSD ardış1k farkların standart sapması ve SD ise verinin standart sapmasıdır.

$$
\begin{gathered}
S D_{1}=\sqrt{\frac{1}{2} S D S D^{2}}=\operatorname{std}\left(\frac{x_{i+1}-x_{i}}{\sqrt{2}}\right) \\
S D_{2}=\sqrt{2(S D)^{2}-\frac{1}{2} S D S D^{2}}=\operatorname{std}\left(\frac{x_{i+1}+x_{i}}{\sqrt{2}}\right)
\end{gathered}
$$

\section{3. İstatistiksel Tabanlı Öznitelik Seçimi}

Tek yönlü varyans analizi (ANOVA), ikiden fazla bağımsız grubun ortalamaları arasında istatistiksel olarak anlamlı bir fark olup olmadığını belirlemek için kullanılır [14]. Bu çalışmada kullanılan prosesteki 20 farklı arıza çeşidi olduğu için, arızalar arasında istatistiksel olarak anlamlı fark gösteren sensörlerin ve özniteliklerin tespitinde ANOVA kullanılmıştır. ANOVA için tüm öznitelikler ve gruplar SPSS yazılımına yüklenmiş ve istatistiksel anlamlıllk düzeyi $\% 5$ seçildiği için 0,05 'den küçük $\mathrm{p}$ (anlamllık) değeri veren öznitelikler seçilmiştir. Tek yönlü ANOVA gruplar arasında anlamlı fark olduğunu verse de hangi gruplar arasında fark olduğunu ve hangileri arasında fark olmadığını vermez [15]. Bunun için post-hoc testleri kullanılır. $\mathrm{Bu}$ çalışmada sadece istatistiksel anlamlı fark gösterdiğini bilmek yeterli olduğundan, post-hoc testleri uygulanmamıştır.

\subsection{Sınıflandırıcı}

Topluluk öğrenmesi (Ensemble learning), birkaç modeli birleştirerek makine öğrenimi sonuçlarının iyileştirilmesine yardımcı olur. Bu yaklaşım, tek bir modele kıyasla daha iyi tahmin performansı üretilmesine olanak tanır. $\mathrm{Bu}$ nedenle topluluk öğrenmesi yöntemleri, Netflix Yarışması, KDD 2009 ve Kaggle gibi birçok makine öğrenimi yarışmasında birinci sırada yer almıştır $[7,8,9]$.

Topluluk öğrenmesi yöntemleri, varyansı (bagging), sapmayı (boosting) azaltmak veya tahminleri iyileştirmek (stacking) için birkaç makine öğrenimi tekniğini tek bir tahmin modelinde birleştiren algoritmalardır. AdaBoost gibi temel öğrenicilerin sıralı olarak üretildiği toplu yöntemler ve Random Forest gibi paralel yöntemler olmak üzere ikiye ayrılır. Sıralı yöntemlerin temel motivasyonu, temel öğrenenler arasindaki bağımlılıktan yararlanmaktır. Performans, önceden yanlış etiketlenmiş örnekleri daha yüksek ağırlıkla tartarak artırılabilir. Paralel yöntemlerin temel motivasyonu, temel öğrenenler arasındaki bağımsızlıktan yararlanmaktır. Sinıflandırıcı genel hatası, öğrenicilerin ortalaması alınarak önemli ölçüde azaltılmaktadır. Çoğu topluluk öğrenmesi yöntemi, homojen temel öğrenenler, yani aynı türden öğrenenler üretmek için tek bir temel öğrenme algoritması kullanır ve bu da homojen topluluklara yol açar [12].

Bu çalışmada kullanılan veri seti, test ve eğitim olmak üzere rasgele seçilen örneklerden oluşan iki gruba ayrılmıştır [13]. Eğitim grubuna ait verilerle sınıflandırıcı eğitimleri yapılırken, test grubu verileriyle sınıflandırıcı başarımları hesaplanmıştır. Bunun için topluluk öğreniminin Boosted Trees, Bagged Trees, Subspace Discriminant, Subspace KNN ve RUSBoosted Trees metotları kullanıldı. Bu metotların performans karşılaş̧ırması Doğruluk (Accuracy) ile yapılmıştır [3].

$$
\text { Doğruluk }=\frac{\text { Doğru sınıflandırılan örnek sayısı }}{\text { Toplam örnek sayısı }}
$$




\subsection{Uygulama}

Tennessee Eastman Process'den alınan veriler, Poincare plot ölçümleri kullanılarak hesaplandı ve SD1 ve SD2 değerlerini içeren bir veri seti oluşturulmuştur. Bu veri seti kullanılarak 20 farklı hatayı sınıflandırmak için Topluluk Öğrenmesi algoritmaları (Boosted Trees, Bagged Trees, Subspace Discriminant, Subspace KNN ve RUSBoosted Trees) koşturulmuştur. Daha sonra, bu veri seti içindeki Poincare ölçümlerinden hangilerinin $\% 5$ anlamlılık düzeyinde hata türleri arasında farklılık gösterdiğini bulmak için tek yönlü ANOVA testi kullanılmıştır. $\mathrm{Bu}$ istatistiksel olarak seçilen Poincare özelliklerinin oluşturduğu yeni veri seti kullanılarak 20 farklı hatayı sınıflandırmak için aynı sınıflandırıcı algoritmaları koşturulmuştur. Tüm sınıflandırıcıların başarımları doğru olarak sınıflandırdıkları hata sayısının tüm hata sayısına oranı olarak formül (3) kullanılarak hesaplanarak karşılaştırılmıştır. Şekil 2'de önerilen arıza tespit sisteminin çalışması özetlenmektedir.

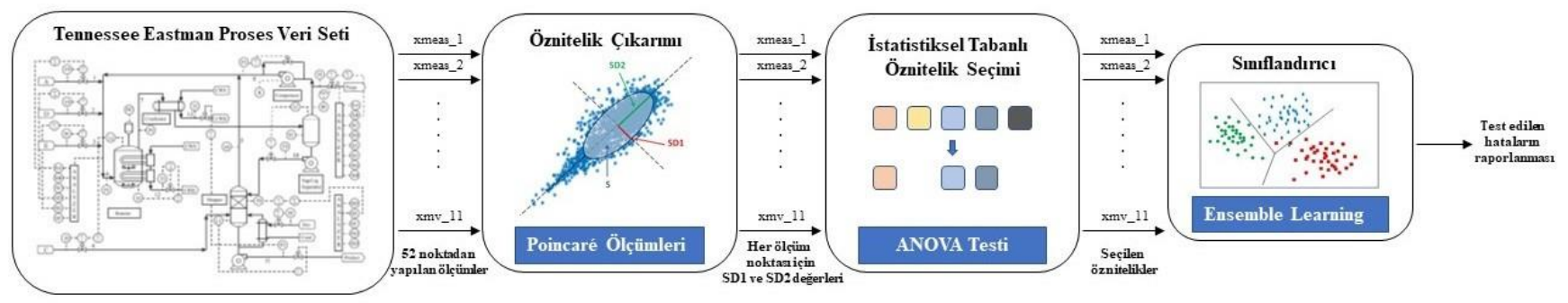

Şekil 2. Önerilen arıza tespit sistem şeması

\section{Sonuçlar}

Bu çalışmada, Poincaré ölçümleri (bir başka deyişle SD1 ve SD2 değerleri) bulundu ve elde edilen veri seti ile ölçümler arasında anlamlı farklılık olanları bulmak için tek yönlü ANOVA testi kullanıldı. Bu test sonucunda 24, 26, 32, 37, 39,
40 ve 41 nolu sensörlere ait SD1 ölçümlerinin istatistiksel olarak anlamlı fark göstermediği tespit edildiğinden, bu öznitelikler veri setinden çıkarılarak yeni bir veri seti oluşturulmuştur. Tüm Poincaré ölçümlerinden ve istatistiksel olarak seçilen Poincaré ölçümlerinden 20 farklı hatayı sinıflandırmak için kullanılan Topluluk Öğrenimi metotları ve doğruluk yüzdeleri Tablo 1'de özetlenmiştir.

Tablo 1. Ham Poincaré ölçümleri ve tek yönlü ANOVA ile seçilmiş Poincaré ölçümlerinin farklı topluluk öğrenim metotlarındaki başarl yüzdeleri

\begin{tabular}{l|c|c}
\hline $\begin{array}{c}\text { Topluluk Öğrenmesi } \\
\text { Algoritmaları }\end{array}$ & $\begin{array}{c}\text { Tüm Özniteliklerin Kullanıldığı } \\
\text { Zaman Sınıflandırıc Doğruluğu }\end{array}$ & $\begin{array}{c}\text { Sadece ANOVA ile Seçilen Özniteliklerin } \\
\text { Kullanıldı̆̆ı Zaman Sınıflandırıcı Doğruluğu }\end{array}$ \\
\hline Boosted Trees & $\% 79,0$ & $\% 81,5$ \\
\hline Bagged Trees & $\% 70,0$ & $\% 70,5$ \\
\hline Subspace Discriminant & $\mathbf{\% 8 9 , 5}$ & $\% \mathbf{8 9 , 5}$ \\
\hline Subspace KNN & $\% 53,5$ & $\% 51,5$ \\
\hline RUSBoosted Trees & $\% 73,0$ & $\% 76,5$ \\
\hline
\end{tabular}

Tablo 1'e göre, Subspace Discriminant algoritması \%89,5 ile en yüksek sınıflandırıcı doğruluğunu vermiştir. Öznitelik seçimi yapılan ve yapılmayan veri setlerine ait sınıflandırıcı

\section{Tartışma}

Arıza tespit ve teşhis teknolojisi endüstriyel proseslerin ve sistemlerin tutarlılığını, dayanıklılığını, güvenliğini sağlamak için en kritik bileşenlerden biridir. Bu çalışmada, hataları henüz oluşmadan tahmin etdilmesi ve tedbir alınması için sensörlerden gelen verilerden hesaplanan Poincare çizimi öznitelikleri üzerinden Topluluk Öğrenmesi tabanlı bir uyarı sistemi geliştirilmiştir (Şekil 2).

$\mathrm{Bu}$ çalışmada hataları tahmin etmek ve sınıflandırmak için en etkili makine öğrenme yöntemlerinden biri olan Topluluk e-ISSN: 2148-2683 algoritmalarının doğrulukları kıyaslandığında, aralarında küçük farklar bulunduğu görülmektedir.

Öğrenmesinin farklı algoritmaları denenmiştir. Bu algoritmalar arasından Subspace Discriminant algoritması \%89,5 sınıflandırıcı doğruluğu ile en yüksek performansı vermiştir. Çok yakın tarihli bir çalışmada, yine Poincaré ölçümleri kullanılarak en yakın k komşuluk (KNN) sınıflandırıcısı ile \%55 sınıflandırıcı doğruluğuna ulaşıldığı görülmektedir [10]. Başka bir başka çalışmada ise destek vektör makineleri (SVM), PCA uygulanmış SVM ve çok katmanlı algılayıcı (MLP) sinıflandırıcıları kullanılarak sirasıyla $\% 49,7, \% 67,3$ ve $\% 73,8$ sınıflandırıcı doğruluklarına ulaşılabilmiştir [11]. Literatürde verilen bu az sayıdaki çalışma ile kıyaslandığında, bu çalışmada 


\section{Avrupa Bilim ve Teknoloji Dergisi}

elde edilen Topluluk Öğrenmesi sınıflandırıcısı en yüksek sınıflandırıcı başarımına ulaşmıştır. Üstelik önceki çalışmalarda, kullanılmayan bir doğrusal olmayan öznitelikler arasında yer alan Poincare Çizimi özniteliklerinin de etkili olduğu görülmektedir. Takip eden çalışmalarda, diğer iyi bilinen makine öğrenmesi algoritmalarının, öznitelik çıkarma ve seçme yöntemlerinin kullanılmasıyla daha yüksek bir sınıflandırıcı performansının elde edilmesi hedeflenmektedir.

\section{Kaynakça}

[1] Chadha, G. S., \& Schwung, A. (2017, September). Comparison of deep neural network architectures for fault detection in Tennessee Eastman process. In 2017 22nd IEEE International Conference on Emerging Technologies and Factory Automation (ETFA) (pp. 1-8). IEEE.

[2] Hajihosseini, P., Anzehaee, M. M., \& Behnam, B. (2018). Fault detection and isolation in the challenging Tennessee Eastman process by using image processing techniques. ISA transactions, 79, 137-146.

[3] D’Angelo, M. F., Palhares, R. M., Camargos Filho, M. C., Maia, R. D., Mendes, J. B., \& Ekel, P. Y. (2016). A new fault classification approach applied to Tennessee Eastman benchmark process. Applied Soft Computing, 49, 676-686.

[4] Downs, J. J., \& Vogel, E. F. (1993). A plant-wide industrial process control problem. Computers \& chemical engineering, 17(3), 245-255.

[5] Ricker, N. L. (1996). Decentralized control of the Tennessee Eastman challenge process. Journal of Process Control, 6(4), 205-221.

[6] Nashalji, M. N., Shoorehdeli, M. A., \& Teshnehlab, M. (2010). Fault detection of the Tennessee Eastman process using improved PCA and neural classifier. In Soft computing in industrial applications (pp. 41-50). Springer, Berlin, Heidelberg.

[7] Puurula, A., Read, J., \& Bifet, A. (2014). Kaggle LSHTC4 winning solution. arXiv preprint arXiv:1405.0546.

[8] Niculescu-Mizil, A., Perlich, C., Swirszcz, G., Sindhwani, V., Liu, Y., Melville, P., ... \& Zhu, Y. F. (2009, December). Winning the KDD cup orange challenge with ensemble selection. In KDD-Cup 2009 Competition (pp. 23-34). PMLR.

[9] Schclar, A., Tsikinovsky, A., Rokach, L., Meisels, A., \& Antwarg, L. (2009, October). Ensemble methods for improving the performance of neighborhood-based collaborative filtering. In Proceedings of the third ACM conference on Recommender systems (pp. 261-264).

[10] Cancioglu, E., Sahin, S., \& Isler, Y. (2021). Fault Detection and Diagnosis on Process Control Systems Using k-Nearest Neighbors from Poincare Plot Measures. In International Conference on Applied Sciences, Engineering and Mathematics (ICASEM'2021), ACCEPTED, Skopje, North Macedonia.

[11] Mireles Gonzalez, J. I. (2018). Deep recurrent neural networks for fault detection and classification (M.Sc. Thesis, University of Waterloo).

[12] Duda, R. O., Hart, P. E. \& Stork, D. G. (2001). Pattern Classification. New York: John Wiley and Sons, 2nd Edition.

[13] Isler, Y., Narin, A. \& Ozer, M. (2015). Comparison of the effects of cross-validation methods on determining performances of classifiers used in diagnosing congestive heart failure. Measurement Science Review, 15(4), 196-201.

[14] Narin, A., Isler, Y. \& Ozer, M. (2014). Investigating the performance improvement of HRV Indices in CHF using feature selection methods based on backward elimination and statistical significance. Computers in Biology and Medicine, 45, 72-79.

[15] Akgul, A. (2003). Tıbbi Araştırmalarda İstatistiksel Analiz Teknikleri: SPSS Uygulamaları. Seçkin Yayıncılık, Ankara, Turkey.

[16] Isler, Y. \& Kuntalp, M. (2007). Combining classical HRV indices with wavelet entropy measures improves to performance in diagnosing congestive heart failure. Computers in Biology and Medicine, 37(10), 1502-1510.

[17] Isler, Y. (2009). A Detailed Analysis of the Effects of Various Combinations of Heart Rate Variability Indices in Congestive Heart Failure. PhD thesis, Department of Electrical and Electronics Engineering, The Graduate School of Natural and Applied Sciences, Dokuz Eylul University.

[18] Marciano, M. L., Migaux, F., Acanfora, D., Furgi, G. \& Rengo, R. (1994). Quantification of poincare maps for the evaluation of heart rate variability. Computers in Caridology, 1994, 557-580.

[19] Brennan, M., Palaniswami, M. \& Kamen, P. (2001). Do existing measures of poincare plot geometry reflect nonlinear features of heart rate variability? IEEE Transactions on Biomedical Engineering, 48(11), 1342-1347.

[20] Chen, X. (2019). Tennessee Eastman simulation dataset. IEEE Dataport, https://dx.doi.org/10.21227/4519-z502. 